\title{
On Oscillatory Instability in Convective Burning of Gas-Permeable Explosives
}

\author{
I. Brailovsky ${ }^{1}$, M. Frankel $^{2}$, L. Kagan ${ }^{1}$ and G. Sivashinsky ${ }^{1}$ * \\ ${ }^{1}$ School of Mathematical Sciences, Tel Aviv University, Ramat Aviv 69978, Israel \\ ${ }^{2}$ Department of Mathematical Sciences, Indiana University - Purdue University, \\ Indianapolis, IN 46202, USA
}

\begin{abstract}
The experimentally known phenomenon of oscillatory instability in convective burning of porous explosives is discussed. A simple phenomenological model accounting for the ejection of unburned particles from the consolidated charge is formulated and analyzed. It is shown that the post-front hydraulic resistance induced by the ejected particles provides a mechanism for the oscillatory burning.
\end{abstract}

Key words: gas-permeable explosives, convective burning, oscillatory instability

AMS subject classification: $80 \mathrm{~A} 32,92 \mathrm{E} 20$

\section{Introduction}

This paper is concerned with the oscillatory instability often observed in high-velocity convective burning of gas-permeable solid explosives $[1,2,4,9,10]$. In convective burning preheating of the porous charge is effected by convective transport of hot gases driven by the developing pressure gradients. The latter, in turn, are induced by the resistance of the gas flow in the interstices between the explosive particles. In the course of burning the solid explosive converts into gaseous products which results in complete suppression of hydraulic resistance. In practical situations the solidto-gas conversion is often accompanied by ejection of unburned particles from the consolidated charge. This results in an extension of both reaction- and resistance-zones behind the regressing surface (front) of the charge [12] (Fig. 1). As we intend to show, precisely this post-front resistance

*Corresponding author. E-mail: grishas@post.tau.ac.il 
provides a mechanism for the oscillatory burning.

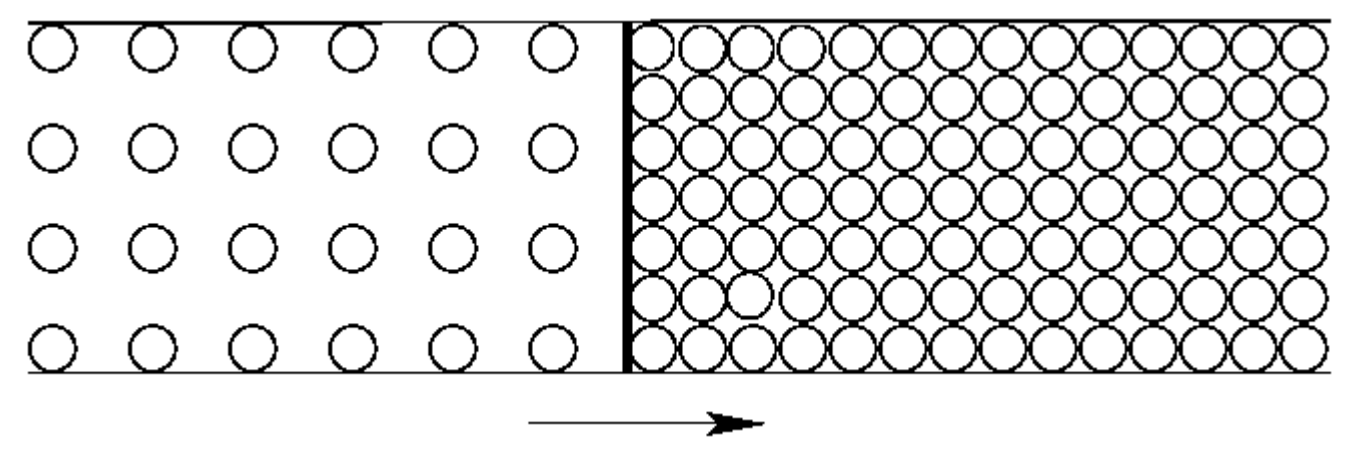

Figure 1: Schematic diagram of combustion wave corresponding to the present model. Arrow indicates direction of propagation. Bold line represents the reaction front. The right/left side of the front corresponds to the unburned/partially-burned solid.

It should be noted that the low-velocity conductive burning may also occur in an oscillatory mode (Telengator et al [16]). In this case, however, the reaction spread is controlled by the system's thermal conductivity, while the impact of hydraulic resistance is negligibly small.

\section{Formulation}

The model employed here is basically a slight modification of the formulation adopted in Telengator, Williams and Margolis [17]. Specifically, we assume that the bulk of heat release occurs at the leading front (Fig. 1) while the role of ejected particles is merely to provide some postfront hydraulic resistance. To reduce the number of parameters involved the 'burn out' distance of the ejected particles is assumed to be much wider than the precompression zone adjacent to the front. The ejected particles therefore are perceived as a residual solid phase whose volume fraction is regarded as prescribed. The gas and solid are assumed to be in thermal equilibrium, allowing utilization of a single-temperature description for the two-phase medium. The solid phase is considered to be firmly fixed in space. The reaction-rate is specified by a one-step Arrhenius kinetics converting the solid explosive straightforwardly into the gaseous product with the solid 'sediment'. The gas-phase volume fraction (porosity) serves as the reaction progress-variable. The reaction terminates as the porosity approaches some prescribed value $\phi_{\text {res }}$. As an equation of state a perfect gas law is used. The classical Darcy's law is employed as a momentum equation.

The current study is focused on the subsonic propagation only. Moreover, we consider the limit of zero-Mach-number where the effects due to dynamic compressibility are ignored.

In the high-velocity convective burning the impact of thermal conductivity is negligibly week compared to convective transport. The pertinent ingredients in the energy balance may therefore be suppressed. The set of governing equations may thus be written as follows. 
Mass conservation for gaseous phase,

$$
\frac{\partial(\phi \rho)}{\partial t}+\frac{\partial(\phi \rho u)}{\partial x}=\rho_{s} \frac{\partial \phi}{\partial t}
$$

Momentum equation (Darcy's law),

$$
\phi u=-\frac{K}{\mu} \frac{\partial P}{\partial x}
$$

Energy conservation,

$$
\frac{\partial}{\partial t}\left[(1-\phi) c_{s} \rho_{s} T+\phi c_{p} \rho T\right]+\frac{\partial}{\partial x}\left(\phi c_{p} \rho u T\right)=\phi \frac{\partial P}{\partial t}+Q \rho_{s} \frac{\partial \phi}{\partial t}
$$

Equation of state,

$$
P=\left(c_{p}-c_{v}\right) \rho T
$$

Solid phase conservation,

$$
\rho_{s} \frac{\partial \phi}{\partial t}=\frac{\rho_{s}\left(\phi_{\text {res }}-\phi\right)}{\tau_{r}}
$$

Here $T, P, \rho, u$ and $\phi$ are the temperature, pressure, gas density, flow velocity and porosity, respectively. $\rho_{s}$ is the density of solid phase. $c_{p}, c_{v}, c_{s}$ are specific heats for gaseous and solid phases. $\mu$ is the dynamic viscosity. Specific heats and viscosity are assumed to be constant. $\tau_{r}$ is the reaction-time defined by the Arrhenius temperature-dependence,

$$
\frac{1}{\tau_{r}}=A \exp \left(-\frac{T_{a}}{T}\right)
$$

where $T_{a}$ is the activation temperature, and $A$ is the Arrhenius prefactor, assumed to be constant. $K$ is the permeability, which here is specified by the Ergun correlation [11],

$$
K=\frac{\phi^{3} d_{p}^{2}}{150(1-\phi)^{2}},
$$

where $d_{p}$ is the solid particle diameter.

The system of Equations (2.1) - (2.7) constitutes a closed set for variables $T, P, \rho, u$ and $\phi$. The equations are complimented by the boundary conditions,

$$
\begin{gathered}
T \rightarrow T_{0}, \quad P \rightarrow P_{0}, \quad \rho \rightarrow \rho_{0}=P_{0} /\left(c_{p}-c_{v}\right) T_{0}, \quad u \rightarrow 0, \quad \phi \rightarrow \phi_{0}, \quad \text { as } \quad x \rightarrow+\infty \\
\partial T / \partial x \rightarrow 0, \quad \partial P / \partial x \rightarrow 0, \quad \partial \rho / \partial x \rightarrow 0, \quad u \rightarrow 0, \quad \phi \rightarrow \phi_{\text {res }}, \quad \text { as } \quad x \rightarrow-\infty
\end{gathered}
$$

Conditions (2.8) (2.9) correspond to the burning under complete confinement.

To make the stability problem tractable analytically, and hopefully without much loss to the essential physics involved, in further discussion we adopt the quasi-linear, high-porosity $\phi \simeq 1$ 
approximation, where variations of porosity, temperature, pressure, density, and flow-velocity are assumed to be small. As a result the nonlinear effects may be ignored everywhere but in the reaction-rate and permeability terms, which are highly sensitive to even minor changes in temperature and porosity. Thus we set, $T=T_{0}+\delta T, P=P_{0}+\delta P, \rho=\rho_{0}+\delta \rho, \phi=\phi_{0}+\delta \phi, u=\delta u$, where $P_{0}, T_{0}, \rho_{0}, \phi_{0}$ correspond to the initial state of the system prior to ignition (see (2.8)).

Upon partial linearization, assuming $\delta$-terms to be small, $1-\phi_{0} \sim \phi_{\text {res }}-\phi_{0} \sim \delta \phi$, and $\rho_{s}>>\rho_{0}$, Eqs. (2.1) - (2.9) yield,

$$
\begin{gathered}
\frac{\partial(\delta \rho)}{\partial t}+\rho_{0} \frac{\partial(\delta u)}{\partial x}=\rho_{s} \frac{\partial(\delta \phi)}{\partial t} \\
\delta u=-\frac{K}{\mu} \frac{\partial(\delta P)}{\partial x} \\
\rho_{0} c_{p} \frac{\partial(\delta T)}{\partial t}=\frac{\partial(\delta P)}{\partial t}+Q_{*} \rho_{s} \frac{\partial(\delta \phi)}{\partial t} \\
Q_{*}=Q+\left(c_{s}-c_{p}\right) T_{0} \\
\delta \rho=\left(\rho_{0} / P_{0}\right) \delta P-\left(\rho_{0} / T_{0}\right) \delta T \\
\frac{\partial(\delta \phi)}{\partial t}=\frac{\left(\phi_{\text {res }}-\phi_{0}\right)-\delta \phi}{\tau_{r}} \\
\frac{1}{\tau_{r}}=A \exp \left(-\frac{T_{a}}{T_{0}+\delta T}\right) \\
K=\frac{d_{p}^{2}}{150\left(\left(1-\phi_{0}\right)-\delta \phi\right)^{2}} \\
\delta(\delta T) / \partial x \rightarrow 0, \partial(\delta P) / \partial x \rightarrow 0, \quad \partial(\delta \rho) / \partial x \rightarrow 0, \delta u \rightarrow 0, \delta \phi \rightarrow \phi_{\text {res }}-\phi_{0}, \text { as } x \rightarrow-\infty
\end{gathered}
$$

Equation (2.12) and boundary conditions (2.17) readily imply a useful relation between $\delta T, \delta P$, and $\delta \phi$,

$$
\delta T=\frac{1}{c_{p} \rho_{0}}(\delta P)+\frac{Q_{*} \rho_{s}}{c_{p} \rho_{0}}(\delta \phi)
$$

By virtue of Eqs. (2.11) (2.13) the mass conservation equation (2.10) may be written as

$$
\frac{\partial(\delta P)}{\partial t}=\frac{\partial}{\partial x}\left(\frac{\gamma P_{0} K}{\mu} \frac{\partial(\delta P)}{\partial x}\right)+P_{0}\left(\frac{\rho_{s}}{\rho_{0}}\right)\left(1+\frac{Q_{*}}{c_{p} T_{0}}\right) \frac{\partial(\delta \phi)}{\partial t}
$$

The overall dynamical system thus is now governed by a coupled set of differential equations (2.14)(2.20) for $\delta P$ and $\delta \phi$ only. 


\section{Combustion wave}

For a travelling-wave solution $\left(\delta P=\delta P\left(x-U_{\infty} t\right), \delta \phi=\delta \phi\left(x-U_{\infty} t\right)\right)$ Eqs. (2.14)(2.20) yield,

$$
\begin{gathered}
-U_{\infty} \frac{d(\delta P)}{d \xi}=\frac{d}{d \xi}\left(\frac{\gamma P_{0} K}{\mu} \frac{d(\delta P)}{d \xi}\right)-U_{\infty} P_{0}\left(\frac{\rho_{s}}{\rho_{0}}\right)\left(1+\frac{Q_{*}}{c_{p} T_{0}}\right) \frac{d(\delta \phi)}{d \xi} \\
-U_{\infty} \frac{d(\delta \phi)}{d \xi}=\frac{\left(\phi_{r e s}-\phi_{0}\right)-\delta \phi}{\tau_{r}}
\end{gathered}
$$

where $\xi=x-U_{\infty} t$.

Upon integration of Eq. (3.1), subject to the upstream boundary conditions (2.17), one ends up with the following first-order differential equation,

$$
\frac{\gamma P_{0} K}{\mu U_{\infty}} \frac{d(\delta P)}{d \xi}=P_{0}\left(\frac{\rho_{s}}{\rho_{0}}\right)\left(1+\frac{Q_{*}}{c_{p} T_{0}}\right) \delta \phi-\delta P
$$

Hence, for the burned gas pressure (see (2.18)) one obtains,

$$
(\delta P)_{\infty}=P_{0}\left(\frac{\rho_{s}}{\rho_{0}}\right)\left(1+\frac{Q_{*}}{c_{p} T_{0}}\right)\left(\phi_{r e s}-\phi_{0}\right)
$$

Then, by virtue of Eq. (2.19), for the burned gas temperature,

$$
(\delta T)_{\infty}=\frac{1}{c_{p} \rho_{0}}(\delta P)_{\infty}+\frac{Q_{*} \rho_{s}}{c_{p} \rho_{0}}\left(\phi_{r e s}-\phi_{0}\right) .
$$

Across the reaction zone the reduced pressure $\delta P$ is continuous and equal to $(\delta P)_{\infty}$.

Hence, according to Eq. (2.19), at the unburned side of the reaction zone where $\delta \phi=0$,

$$
(\delta T)_{i}=\frac{1}{c_{p} \rho_{0}}(\delta P)_{\infty}
$$

which will be referred to as the 'ignition' temperature. It is precisely the ignition temperature $(\delta T)_{i}$ which controls the propagation velocity $U_{\infty}$ [14]. More specifically, at high activation energies, $T_{a}>T_{0}$, the burning rate is determined by the reaction occurring within a thin layer where

$$
\delta T /(\delta T)_{i}-1 \sim \delta P /(\delta P)_{\infty}-1 \sim T_{0} / T_{a}
$$

Hence, the Arrhenius exponent in Eq. (2.15) may be approximated as (see Eqs. (2.19), (3.6)) and Ref.[14]).

$$
\begin{gathered}
\exp \left(-\frac{T_{a}}{T_{0}+\delta T}\right) \simeq \exp \left[-\frac{T_{a}}{T_{0}+(\delta T)_{i}}\right] \exp \left[\frac{T_{a}\left(\delta T-(\delta T)_{i}\right)}{\left(T_{0}+(\delta T)_{i}\right)^{2}}\right]= \\
\exp \left[-\frac{T_{a}}{T_{0}+(\delta T)_{i}}\right] \exp \left[\frac{T_{a}\left(\delta P-(\delta P)_{\infty}\right)+Q_{*} \rho_{s}(\delta \phi)}{c_{p} \rho_{0}\left(T_{0}+(\delta T)_{i}\right)^{2}}\right]
\end{gathered}
$$


To evaluate $U_{\infty}$ it is helpful to eliminate the spatial variable $\xi$, thereby converting Eqs.(3.1)(3.2) into a single equation relating $d(\delta P)$ and $d(\delta \phi)$. By virtue of (3.7) (3.8) the latter may be written as,

$$
\begin{gathered}
\frac{\gamma A K_{0} P_{0}\left(\phi_{\text {res }}-\phi_{0}\right)}{\mu(\delta P)_{\infty}} \exp \left[-\frac{T_{a}}{T_{0}+(\delta T)_{i}}\right] \exp \left[-\frac{T_{a}\left(\delta P-(\delta P)_{\infty}\right)}{c_{p} \rho_{0}\left(T_{0}+(\delta T)_{i}\right)^{2}}\right] d(\delta P)= \\
U_{\infty}^{2} \exp \left[-\frac{T_{a} Q_{*} \rho_{s}(\delta \phi)}{c_{p} \rho_{0}\left(T_{0}+(\delta T)_{i}\right)^{2}}\right] d(\delta \phi),
\end{gathered}
$$

where

$$
K_{0}=\frac{d_{p}^{2}}{150\left(1-\phi_{0}\right)^{2}}
$$

The right of Eq. (3.9) thus depends only on $\delta \phi$, and the left- only on $\delta P$.

The global integration of Eq. (3.9) over $0<\delta \phi<\phi_{\text {res }}-\phi_{0}$ and $0<\delta P<(\delta P)_{\infty}$, then readily yields,

$$
U_{\infty}^{2}=\frac{\gamma A K_{0} P_{0} Q_{*} \rho_{s}\left(\phi_{\text {res }}-\phi_{0}\right)}{\mu(\delta P)_{\infty}} \exp \left[-\frac{T_{a}}{T_{0}+(\delta T)_{i}}\right]
$$

Here $(\delta T)_{i}$, is related to the external pressure $(\delta P)_{\infty}$, according to Eq. (3.6).

At high activation energies $\left(T_{a}>>T_{0}\right)$ the reaction zone shrinks, turning the reaction rate term on the right of Eq. (3.2) into a localized source $\left(\phi_{\text {res }}-\phi_{0}\right) U_{\infty} \delta(\xi)$.

The above analysis deals with the steady travelling wave solution. One may expect however, that at high activation energies Eq. (3.11) is valid also for the unsteady wave, provided the term $(\delta P)_{\infty}$ in the exponent of Eq. (3.11) (see Eq.(3.6)) is replaced by $\delta P$, the pressure at the wave front, $x=R(t)$. Equation (2.14) may thus be written as

$$
\frac{\partial(\delta \phi)}{\partial t}=\left(\phi_{r e s}-\phi_{0}\right) U \delta(x-R(t))
$$

Here $d R / d t=U$ and $U=U_{\infty} I$ where,

$$
I=\exp \left[\frac{c_{p} \rho_{0} T_{a}\left(\delta P-(\delta P)_{\infty}\right)}{2\left(c_{p} \rho_{0} T_{0}+(\delta P)_{\infty}\right)\left(c_{p} \rho_{0} T_{0}+\delta P\right)}\right]
$$

\section{Nondimensionalization}

To tackle the stability problem both analytically and numerically it is convenient to deal with its nondimensional formulation. The scaled variables are defined as follows.

$\Pi=\delta P /(\delta P)_{\infty}$ - scaled pressure, $\Phi=\left(\left(\phi_{\text {res }}-\phi_{0}\right)-\delta \phi\right) /\left(\phi_{\text {res }}-\phi_{0}\right)$ - scaled solid-phase volume fraction, $\kappa=K / K_{0^{-}}$scaled permeability, $\xi=x / x_{\infty}, F=R / x_{\infty}, \tau=t / t_{\infty^{-}}$scaled spatio-temporal co-ordinates, where $x_{\infty}=D_{p} / U_{\infty}, t_{\infty}=D_{p} / U_{\infty}^{2}$ and $D_{p}=\gamma P_{0} K_{0} / \mu$ is the 
pressure-diffusivity.

Setting

$$
\begin{gathered}
\Sigma=\frac{c_{p} \rho_{0} T_{0}}{c_{p} \rho_{0} T_{0}+(\delta P)_{\infty}} \quad(\Sigma<1) \\
\alpha=\frac{c_{p} \rho_{0} T_{a}(\delta P)_{\infty}}{2\left(c_{p} \rho_{0} T_{0}+(\delta P)_{\infty}\right)^{2}}, \quad \text { scaled activation energy, } \\
\Delta=\left(1-\phi_{0}\right)^{2} /\left(1-\phi_{\text {res }}\right)^{2} \quad(\Delta>1), \quad \text { scaled post-front permeability }
\end{gathered}
$$

one ends up with the scaled free-interface problem involving only three independent parameters: $\alpha, \Delta$, and $\Sigma$,

$$
\begin{gathered}
\frac{\partial \Pi}{\partial \tau}=\frac{\partial}{\partial \xi}\left(\kappa(\Phi) \frac{\partial \Pi}{\partial \xi}\right)+I(\Pi) \delta(\xi-F(\tau)) \\
\frac{\partial \Phi}{\partial \tau}=-I(\Pi) \delta(\xi-F(\tau))
\end{gathered}
$$

where

$$
\begin{gathered}
I(\Pi)=\exp \left[\frac{\alpha(\Pi-1)}{\Sigma+(1-\Sigma) \Pi}\right] \\
\kappa(\Phi>0)=1 \quad \text { and } \quad \kappa(\Phi=0)=\Delta
\end{gathered}
$$

Equations (4.4) (4.5) are complimented by the conditions,

$$
\begin{gathered}
\Pi \rightarrow 0 \quad \text { as } \quad \xi \rightarrow+\infty \\
\Phi(\xi>F(\tau))=1 \quad \text { and } \quad \Phi(\xi<F(\tau))=0
\end{gathered}
$$

Note that, except for the variable permeability $\kappa(\Phi)$, the model (4.4) - (4.9) is similar to the freeinterface model for gasless combustion where $\Pi, \Phi$ and $\Sigma$ are regarded as the scaled temperature, deficient reactant mass fraction and initial/burned temperature ratio, respectively. In this context the model has been explored extensively in recent years, and there is a sizable volume of analytical and numerical results on its intrinsic dynamics (see, for example, Refs. [13][15] and literature therein).

At $\kappa=1$ the model is also formally identical to that for the pressure-driven subsonic combustion in an inert porous bed filled with an explosive gas [6].

\section{Basic propagation mode and its stability}

For further analysis it is convenient to introduce a co-ordinate system attached to the front. Thus we define,

$$
\eta=\xi-F(\tau)
$$


in terms of which the problem (4.4) - (4.9) becomes

$$
\begin{gathered}
\frac{\partial \Pi_{1}}{\partial \tau}-I\left(\Pi_{0}\right) \frac{\partial \Pi_{1}}{\partial \eta}=\Delta \frac{\partial^{2} \Pi_{1}}{\partial \eta^{2}}, \quad-\infty<\eta<0 \\
\frac{\partial \Pi_{2}}{\partial \tau}-I\left(\Pi_{0}\right) \frac{\partial \Pi_{2}}{\partial \eta}=\frac{\partial^{2} \Pi_{2}}{\partial \eta^{2}}, \quad 0<\eta<+\infty \\
I\left(\Pi_{0}\right)=\exp \left[\frac{\alpha\left(\Pi_{0}-1\right)}{\Sigma+(1-\Sigma) \Pi_{0}}\right]
\end{gathered}
$$

Boundary conditions at $\eta= \pm \infty$,

$$
\Pi_{1}(-\infty, \tau)=1, \quad \Pi_{2}(+\infty, \tau)=0
$$

At $\eta=0$ the following matching conditions are held,

$$
\begin{gathered}
\Pi_{1}(0, \tau)=\Pi_{2}(0, \tau)=\Pi_{0}(\tau) \\
\Delta \frac{\partial \Pi_{1}(0, \tau)}{\partial \eta}-\frac{\partial \Pi_{2}(0, \tau)}{\partial \eta}=I\left(\Pi_{0}\right)
\end{gathered}
$$

As implied by Eq. (4.5), the front velocity $V=d F / d \tau$ is determined by the relation,

$$
V=I\left(\Pi_{0}\right)
$$

The problem (5.1) - (5.7) allows for the basic time-independent solution,

$$
\Pi_{1}^{(b)}=1, \quad \Pi_{2}^{(b)}=\exp (-\eta), \quad \Pi_{0}^{(b)}=1, \quad V^{(b)}=1
$$

The linear stability analysis of (5.9) leads to the following equation for the instability increment $\omega$ $\left(V-V^{(b)} \sim \exp (\omega t)\right)$

$$
\omega(1-\sqrt{1+4 \Delta \omega})-(\alpha+\omega)(1+\sqrt{1+4 \omega})+2 \alpha(1+\omega)=0
$$

For the marginal stability curve ( $\operatorname{Re} \omega=0$ ), Eq. (5.10) yields (Fig. 2),

$$
\alpha=\frac{a_{1}(\Delta)\left[a_{1}(\Delta)+a(\Delta)\right]}{2\left[a_{1}(\Delta)-1\right]}
$$

where

$$
\begin{gathered}
a(\Delta)=\sqrt{\frac{1}{2}\left(1+\sqrt{1+16 \Delta^{2} \Omega^{2}}\right)} \\
a_{1}(\Delta)=\sqrt{\frac{1}{2}\left(1+\sqrt{1+16 \Omega^{2}}\right)} \\
\Omega^{2}=(\operatorname{Im} \omega)^{2}=\frac{a_{1}^{2}(\Delta) a(\Delta)\left[a_{1}(\Delta)+a(\Delta)\right]}{4\left[\Delta a_{1}(\Delta)+a(\Delta)\right]}
\end{gathered}
$$



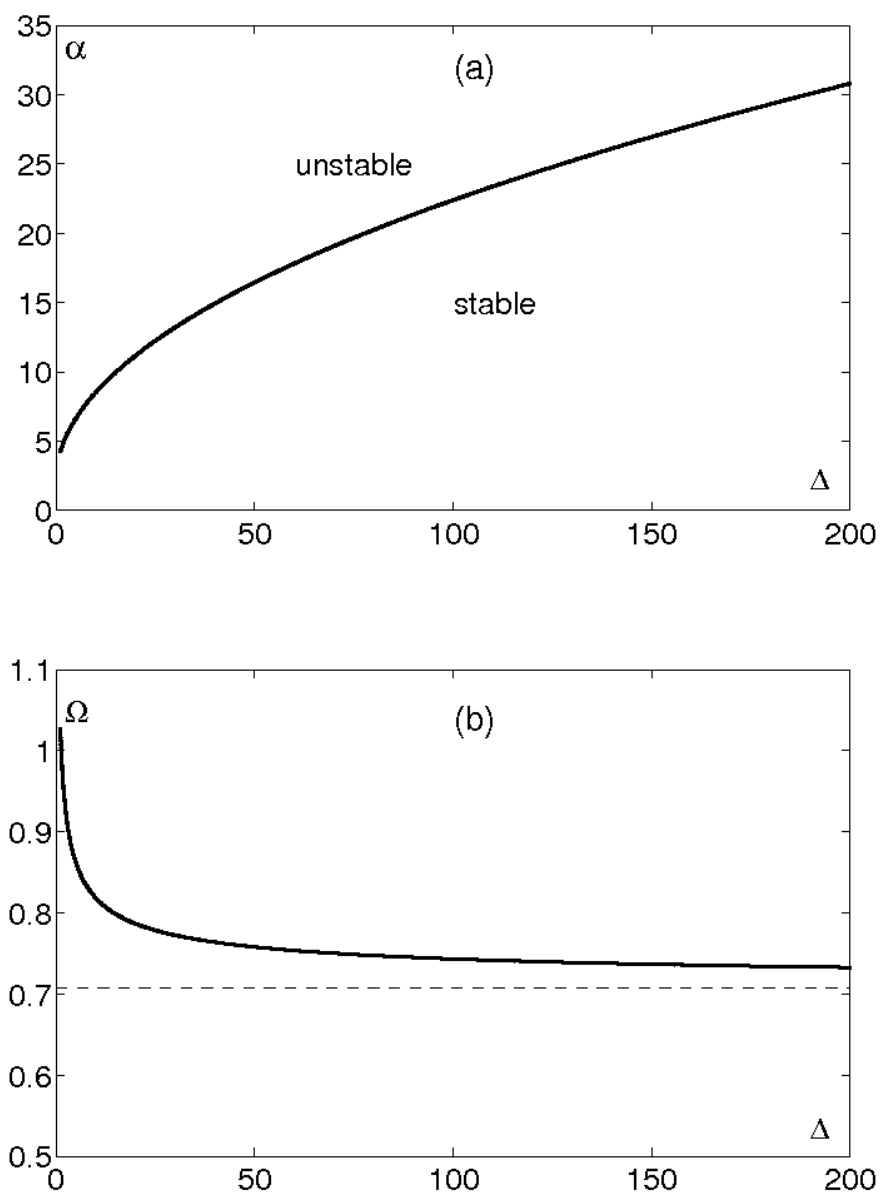

Figure 2: Scaled activation energy (a) and oscillation-frequency (b) vs. scaled post-front permeability at the stability threshold.

Along the marginal stability curve (5.11), $\operatorname{Im} \omega \neq 0$, and hence the passage from the stability to instability domain involves a Hopf-type bifurcation, indicating transition to a time-dependent oscillatory solution.

As may be easily checked,

$$
\begin{aligned}
& \alpha=2+\sqrt{5}, \quad \Omega=\frac{\sqrt{2+\sqrt{5}}}{2} \text { at } \Delta=1, \quad \text { and } \\
& \alpha \simeq(1+\sqrt{2}) \sqrt{\frac{\Delta \sqrt{2}}{2}}, \quad \Omega \simeq \frac{\sqrt{2}}{2} \text { at } \Delta>>1
\end{aligned}
$$

Thus, the stability range expands with the increasing post-front permeability. In the absence of ejected particles $(\Delta=\infty)$, the oscillatory instability is ruled out.

Figures $3-6$ show some results on numerical simulation of the non-linear problem (5.1) - (5.8) under the initial condition $\Pi(\eta, 0)=\Pi^{(b)}(\eta)$. As one might anticipate, close to the stability threshold 
(5.11), the emerging oscillations are periodic (Figs. 4, 5b, 6a). However, deeper in the instability domain the oscillations undergo period doubling (Fig. 6b) eventually assuming an irregular aperiodic structure (Figs. 6c $-6 \mathrm{e}$ ) akin to those occurring in related reaction-diffusion systems $[3,6,8,13,15,16]$.

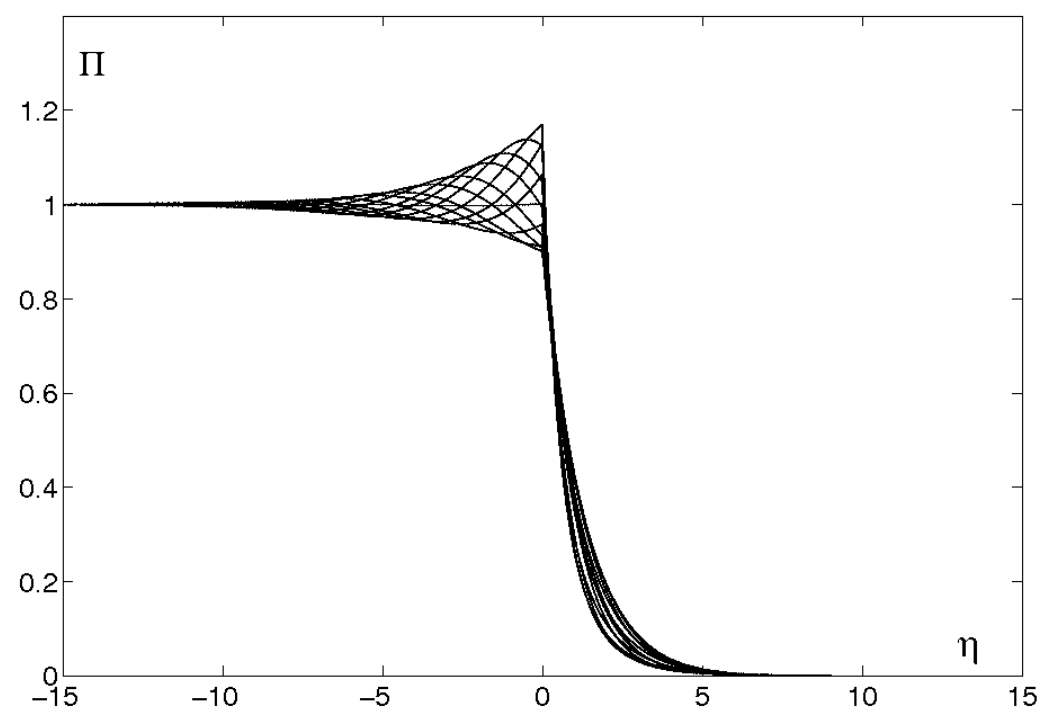

Figure 3: Profiles of pressure at several consecutive instants of time ( $\Delta=1, \alpha=4.4)$. In all simulations $\Sigma$ is set at 0.466 [6].

\section{Concluding remarks}

Although it makes for an analyzable asymptotic limit, the limiting case of high porosity, implying weak perturbations of temperature, pressure and density, would not appear to be an especially realistic regime. Yet, we believe (from previous experience) that the quasi-linear model (4.4)-(4.7) captures correctly the basic 'topology' of the original system (2.1)-(2.7).To validate the model a direct numerical simulation of the fully nonlinear model would be useful.

It would be desirable to put the above model on a more solid physical foundation based on a firstprinciple picture of the formation and subsequent burn-up of the ejected particles.

There are also alternative possibilities for the oscillatory burning worth examining. According to some observations the oscillatory burning is prone to occur in flexible tubes, being suppressed under rigid confinement $[9,10]$. Indeed, it is well known that flexibility of the boundary may exert a destabilizing influence on confined flows [5].

Being controlled by adiabatic compression, the convective burning may be regarded as a variety of low-velocity detonation. The conventional high-velocity detonation often occurs in an oscillatory 


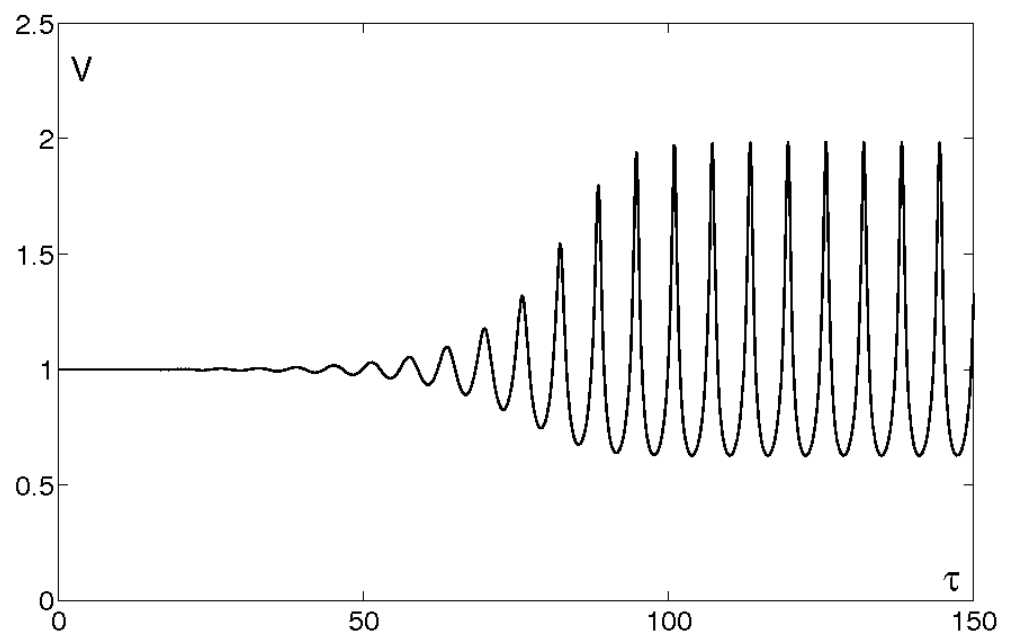

Figure 4: Incipient and fully developed dynamics of the reaction wave velocity $(\Delta=1, \alpha=4.4$ ).

(galloping) mode induced by the thermo-acoustic interaction [7]. It would therefore be interesting to explore this avenue also in the context of convective burning, by abandoning the zero-Machnumber limit and allowing for the dynamic-compressibility effects.

\section{Acknowledgements}

This research has been supported by the US-Israel Binational Science Foundation (Grant 2006151) and the Israel Science Foundation (Grant 32/09).

\section{References}

[1] K. K. Andreev, S. V. Chuiko. Transition of the burning of explosives into an explosion. Russ. J. Phys. Chem., 37 (1963), 695-699.

[2] K. K. Andreev, A. F. Belyaev. Theory of Explosive Substances. Transi., US Department of Commerce Report AD-643597 (1966).

[3] A. Bayliss, B. Matkowsky. Two Routes to Chaos in Condensed Phase Combustion. SIAM J. Appl. Math., 50 (1990), 437-59.

[4] A. F. Belyaev, V. K. Bobolev, A. I. Korotkov, A. A. Sulimov, S. V. Chuiko. Transition from Deflagration to Detonation in Condensed Phases. Israel Program for Scientific Translations, Jerusalem (1975). 

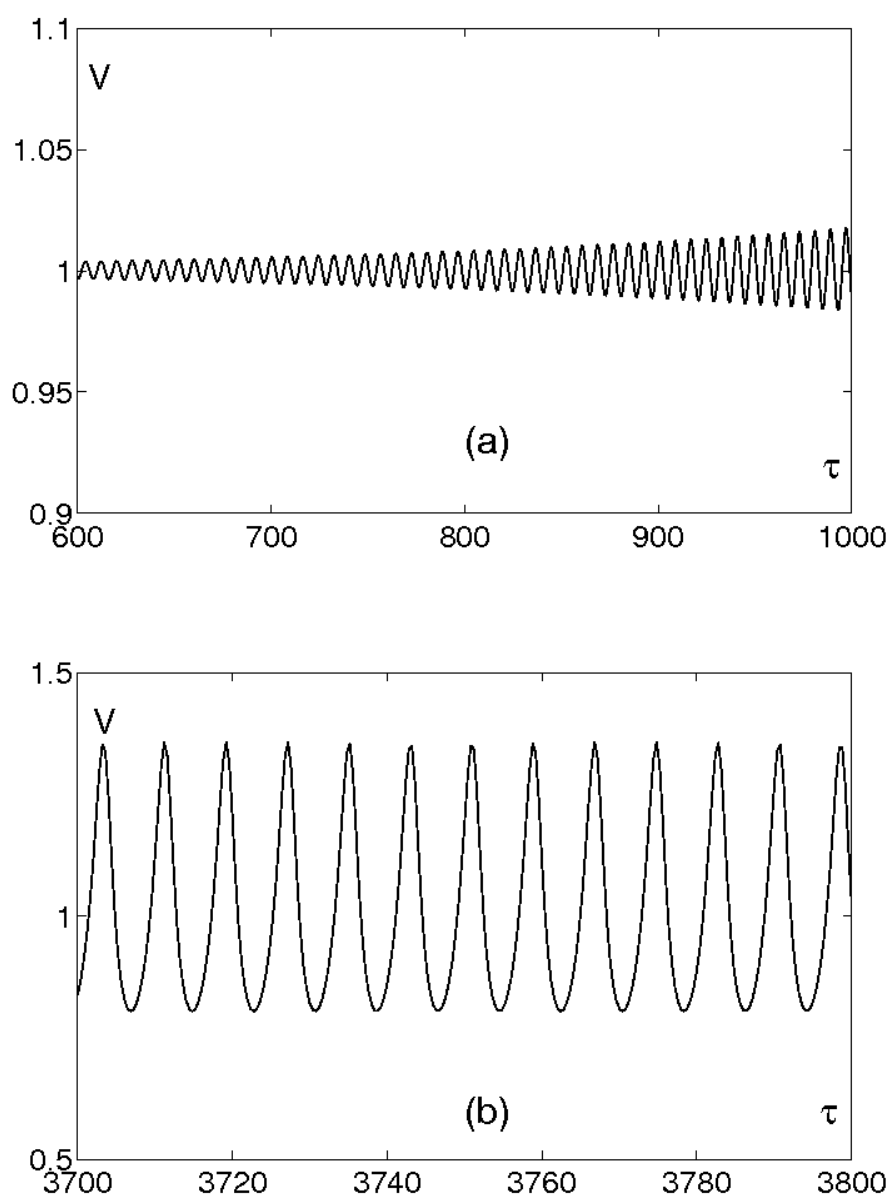

Figure 5: Incipient (a) and well-settled (b) dynamics of the reaction wave velocity ( $\Delta=15$, $\alpha=10)$.

[5] T. B. Benjamin. Effects of a flexible boundary on hydrodynamic stability. J. Fluid Mechanics, 9 (1960), 513-532.

[6] I. Brailovsky, M. Frankel, G. Sivashinsky. Galloping and spinning modes of subsonic detonation. Combust. Theory Modelling, 4 (2000), 47-60.

[7] P. Clavin. Theory of gaseous detonations. Chaos, 14 (2004), 825-38.

[8] P. Dimitriou, J. Puszynski, V. Hlavacek. On the Dynamics of Equations Describing Gasless Combustion in Condensed Systems. Combsut. Sci. Technol., 68 (1989), 101-11.

[9] V. F. Dubovitskii, V. G. Korostelev, A. I. Korotkov, Yu. V. Frolov, A. N. Firsov, K. G. Shkadinsky, S. V. Khomik. Burning of porous condensed systems and powders. Combust.Expl. Shock Waves, 10 (1974), 730-736. 
(a)

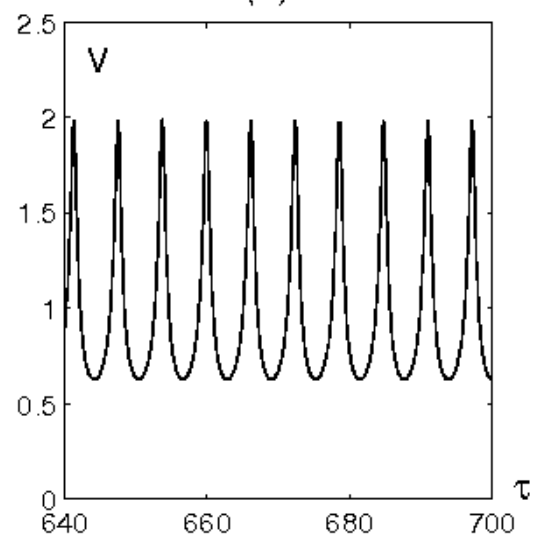

(d)

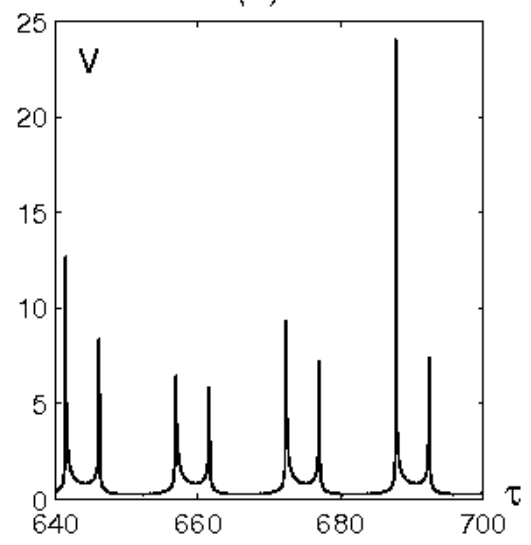

(b)

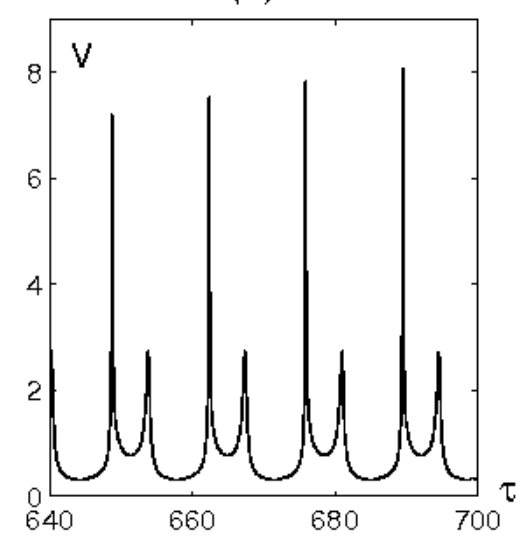

(e)

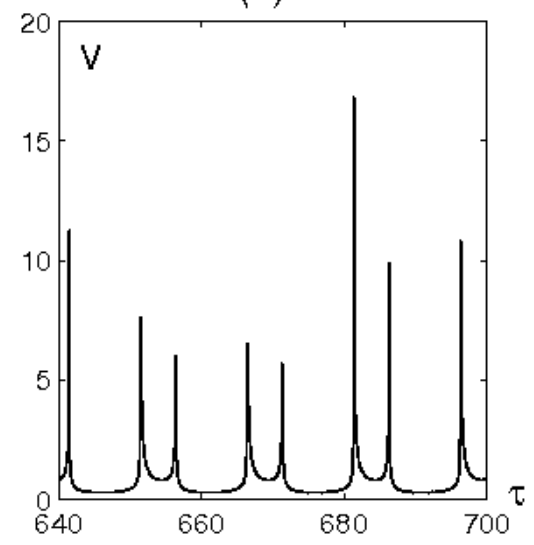

(c)

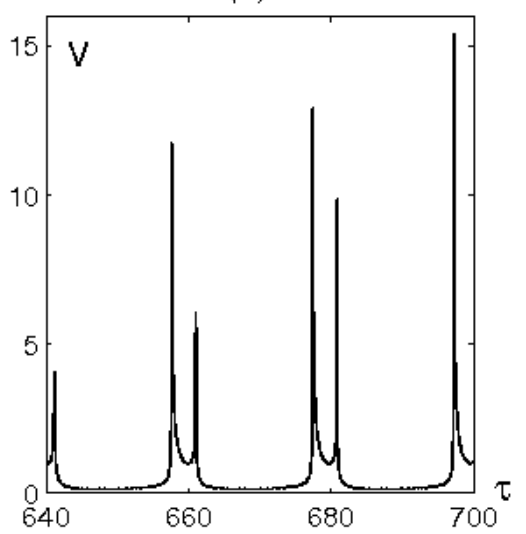

(f)

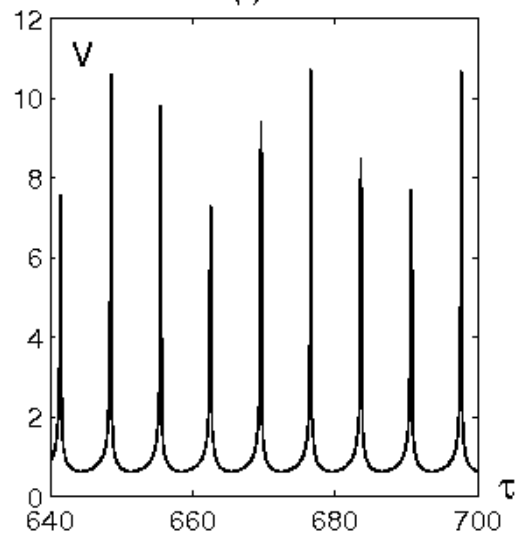

Figure 6: Reaction wave dynamics calculated for $\Delta=1, \alpha=4.4,(a) ; \Delta=1, \alpha=5,(b) ; \Delta=1$, $\alpha=6,(c) ; \Delta=1.5, \alpha=5,(d) ; \Delta=2, \alpha=6.5,(e) ; \Delta=13, \alpha=10,(f)$.

[10] B. S. Ermolaev, A. A. Sulimov, V. A. Foteenkov, V. E. Khrapovskii, A. I. Korotkov, A. A. Borisov. Nature of and laws governing quasi-steady-state pulsed convective combustion. Combust. Expl. Shock Waves, 16 (1980), 266-274.

[11] S. Ergun. Fluid flow through packed columnes. Chem. Engr. Prog. 48 (1952), 89-94.

[12] R. A. Fifer, F. F. Cole. Transition from laminar burning for porous crystalline explosives. Proc. Seventh Symp. (Int.) on Detonation, 7 (1981), 164-174.

[13] M. Frankel, V. Roytburd, G. Sivashinsky. Complex dynamics generated by a sharp interface model of self-propagating high-temperature synthesis. Combust. Theory Modelling, 2 (1998), 479-96. 
[14] L. Kagan, G. Sivashinsky. A high-porosity limit for the transition from conductive to convective burning in gas-permeable explosives. Combust.Flame, 157 (2010), 357-362.

[15] S. B. Margolis. The transition to nonsteady deflagration in gasless combustion. Prog. Energy Combust. Sci., 17 (1991), 135-62.

[16] A. M. Telengator, S. B. Margolis, F. A. Williams. Stability of Quasi-Steady Deflagrations in Confined Porous Energetic Materials. Combust. Sci.Technol., 160 (2000), 259-316.

[17] A. M. Telengator, F. A. Williams, S. B. Margolis. Finite-rate interphase heat-transfer effects on multiphase burning in confined porous propellants. Combust. Sci. Technol., 178 (2006), 1685-1720. 